\title{
Limitations of ultra-thin transparent conducting oxides for integration into plasmonic-enhanced thin-film solar photovoltaic devices
}

\author{
Jephias Gwamuri ${ }^{1}$ - Ankit Vora ${ }^{2}$ Rajendra R. Khanal ${ }^{3}$ - Adam B. Phillips ${ }^{3}$. \\ Michael J. Heben ${ }^{3}$ - Durdu O. Guney ${ }^{2}$ - Paul Bergstrom ${ }^{2}$ - Anand Kulkarni ${ }^{2}$ • \\ Joshua M. Pearce ${ }^{1,2}$ (i)
}

Received: 20 April 2015/Accepted: 3 July 2015/Published online: 16 July 2015

(C) The Author(s) 2015. This article is published with open access at Springerlink.com

\begin{abstract}
This study investigates ultra-thin transparent conducting oxides (TCO) of indium tin oxide (ITO), aluminum-doped zinc oxide (AZO) and zinc oxide $(\mathrm{ZnO})$ to determine their viability as candidate materials for use in plasmonic-enhanced thin-film amorphous silicon solar photovoltaic (PV) devices. First a sensitivity analysis of the optical absorption for the intrinsic layer of a nano-disk patterned thin-film amorphous silicon-based solar cell as a function of TCO thickness $(10-50 \mathrm{~nm})$ was performed by simulation. These simulation results were then used to guide the design of the experimental work which investigated both optical and electrical properties of ultra-thin (10 $\mathrm{nm}$ on average) films simultaneously deposited on both glass and silicon substrates using conventional rf sputtering. The effects of deposition and post-processing parameters on material properties of ITO, AZO and $\mathrm{ZnO}$ ultrathin TCOs were probed and the suitability of TCOs for integration into plasmonic-enhanced thin-film solar PV devices was assessed. The results show that ultra-thin TCOs present a number of challenges for use as thin top contacts on plasmonic-enhanced PV devices: (1) optical and electrical parameters differ greatly from those of
\end{abstract}

Joshua M. Pearce

pearce@mtu.edu

1 Department of Materials Science and Engineering, Michigan Technological University, 1400 Townsend Dr., Houghton, MI 49931-1295, USA

2 Department of Electrical and Computer Engineering, Michigan Technological University, 1400 Townsend Dr., Houghton, MI 49931-1295, USA

3 Wright Center for Photovoltaic Innovation and Commercialization, Department of Physics and Astronomy, School of Solar and Advanced Renewable Energy, University of Toledo, Toledo, $\mathrm{OH} 43606$, USA thicker (bulk) films deposited under the same conditions, (2) the films are delicate due to their thickness, requiring very long annealing times to prevent cracking, and (3) reactive gases require careful monitoring to maintain stoichiometry. The results presented here found a trade-off between conductivity and transparency of the deposited films. Although the sub $50 \mathrm{~nm}$ TCO films investigated exhibited desirable optical properties (transmittance greater than $80 \%$ ), their resistivity was too high to be considered as materials for the top contact of conventional PV devices. Future work is necessary to improve thin TCO properties, or alternative materials, and geometries are needed in plasmonic-based amorphous silicon solar cells. The stability of ultra-thin TCO films also needs to be experimentally investigated under normal device operating conditions.

Keywords Transparent conducting oxide (TCOs) .

Plasmonics $\cdot$ Solar photovoltaics $\cdot$ Indium tin oxide $\cdot$ Zinc oxide $\cdot$ Aluminum-doped zinc oxide

\section{Introduction}

Despite the material, sustainability, economic and technical benefits of thin-film solar photovoltaic (PV) devices [1-3], conventional crystalline silicon (c-Si) modules dominate the market [4]. The cost of c-Si PV has fallen to the point that the balance of systems (BOS) and thus the efficiency of the modules plays a major role in the levelized cost of electricity for solar [5]. There is thus a clear need to improve the efficiency of thin-film devices further [6]. Recent developments in plasmonics theory promise new methods with great potential to enhance light trapping in thin-film PV devices [7-14]. To fully exploit these 
potential benefits offered by plasmonic-based devices, TCOs with high transmittance (low loss) and low enough resistivity are to be used as device top contacts. However, for current transparent conducting oxides (TCOs) to be successfully integrated into the novel proposed plasmonicenhanced PV devices, ultra-thin TCOs films are required [14]. For example, simulations by Vora et al. showed a $19.65 \%$ increase in short circuit current $\left(J_{\mathrm{SC}}\right)$ for nanocylinder patterned solar cell (NCPSC) in which the ITO layer thickness was kept at $10 \mathrm{~nm}$ to minimize the parasitic Ohmic losses and simultaneously act as a buffer layer while helping to tune the resonance for maximum absorption [14]. TCOs such as the most established indium tin oxide (ITO), aluminum-doped zinc oxide (AZO) and zinc oxide $(\mathrm{ZnO})$ are standard integral materials in current thin-film solar PV devices [15-18]. Bulk material properties for common TCOs including ITO have been well researched and documented for different processing conditions and substrates [15, 16, 19-23]; however, this is not the case for ultra-thin TCOs. The few exceptions include Sychkova et al. [24], who reported both optical and electrical properties of 9-80 nm ITO films deposited by pulsed DC sputtering varied with thickness and showed a general increase in resistivity with decrease in film thickness [24]. Other notable studies on ultra-thin ITO films using various deposition techniques include the following: Chen et al. who used filtered cathodic vacuum arc (FCVA) to deposit 30-50 $\mathrm{nm}$ on heated quartz and Si substrates [25]; Tseng and Lo, who used DC magnetron sputter for 34.71-71.64 nm ITO film on PET (polyethylene terephthalate) [26]; Kim et al. who used RF magnetron sputter for films between 40 and $280 \mathrm{~nm}$ deposited on PMMA substrate heated at $70{ }^{\circ} \mathrm{C}$ [27]; Alam and Cameron, who used sol-gel process for $50-250 \mathrm{~nm}$ film deposited on titanium dioxide film [20]; and Betz et al. who used planar DC magnetron sputtering for 50, 100 and $300 \mathrm{~nm}$ films on glass substrates [28]. The results from these few thin TCO studies reveal a pattern in which resistivity increases rapidly as film thickness decreases from 50 to $10 \mathrm{~nm}$.

The electrical properties of ITO thin films depend on the preparation method, the deposition parameters used for a given deposition technique and the subsequent heat treatments. Key factors for the low resistivity have not been clearly documented because of the complex structure of the unit cell of crystalline $\mathrm{In}_{2} \mathrm{O}_{3}$ formed by 80 atoms and the complex nature of the conducting mechanisms in polycrystalline films [29]. The issue is further complicated by the large number of processing parameters, even for a single technique.

To probe these challenges and to determine if ITO, AZO and $\mathrm{ZnO}$ are viable candidate materials for use in plasmonic-enhanced thin-film PV devices, sensitivity analysis on TCO thickness $(10-50 \mathrm{~nm})$ versus absorption was performed using COMSOL Multiphysics RF module v4.3b on the optical absorption in the i-a-Si:H layer of nano-disk patterned thin-film a-Si:H solar cells (NDPSC) shown in Fig. 1a [15]. These simulation results are used to guide the experimental work which investigated both optical and electrical properties of ultra-thin (10 $\mathrm{nm}$ on average) films simultaneously deposited on both glass and silicon substrates (with a thermally grown oxide layer. The effects of deposition and post-processing parameters on material properties of ITO, AZO and ZnO ultra-thin TCOs were probed and the suitability of TCOs for integration into plasmonic-enhanced thin-film solar PV devices was assessed. From these results some of the limitations of thin TCOs for plasmonic optical enhancement of thin-film PV were identified.

\section{The optical effects of TCO thickness}

Sensitivity analysis for the proposed silver nano-disk patterned solar cell (NDPSC) was performed in the $300-750 \mathrm{~nm}$ spectral range to determine the optimum ITO layer thickness which would promote maximum enhancement and minimize Ohmic losses. Having a TCO spacer layer with as low as possible Ohmic losses is desirable for efficient coupling of light from the silver nano-discs into the active layers of the device. The results are shown in Fig. $1 \mathrm{~b}$ and theoretically show $10 \mathrm{~nm}$ films offer the best absorption and hence the greatest potential to improve efficiency in plasmonic-based PV devices. From these results, AZO and ITO offer the best potential due to lower Ohmic losses and $\mathrm{ZnO}$, despite having the greatest Ohmic losses among the three TCOs, is still promising particularly for the sub $20 \mathrm{~nm}$ films since its absorption $\left(>250 \mathrm{~W} / \mathrm{m}^{2}\right)$ is still higher than that expected of a standard PV device.

\section{Experimental details}

The focus of the study was to investigate ways of improving material properties of ultra-thin TCOs for integration into plasmonic-enhanced thin-film solar PV devices by studying the effects of different process parameters on both optical and electrical properties of sub $50 \mathrm{~nm}$ films. A comparative study of the three most commonly used TCOs in thin-film commercial solar cells is undertaken, and a more in-depth study of ITO is performed.

\section{Sample preparation and fabrication}

Samples of ITO with thickness ranging from 10 to $50 \mathrm{~nm}$ were deposited on both glass and n-doped silicon (with a 32-nm thermally grown oxide layer) substrates using rf sputter deposition techniques previously described in refs 
(a)

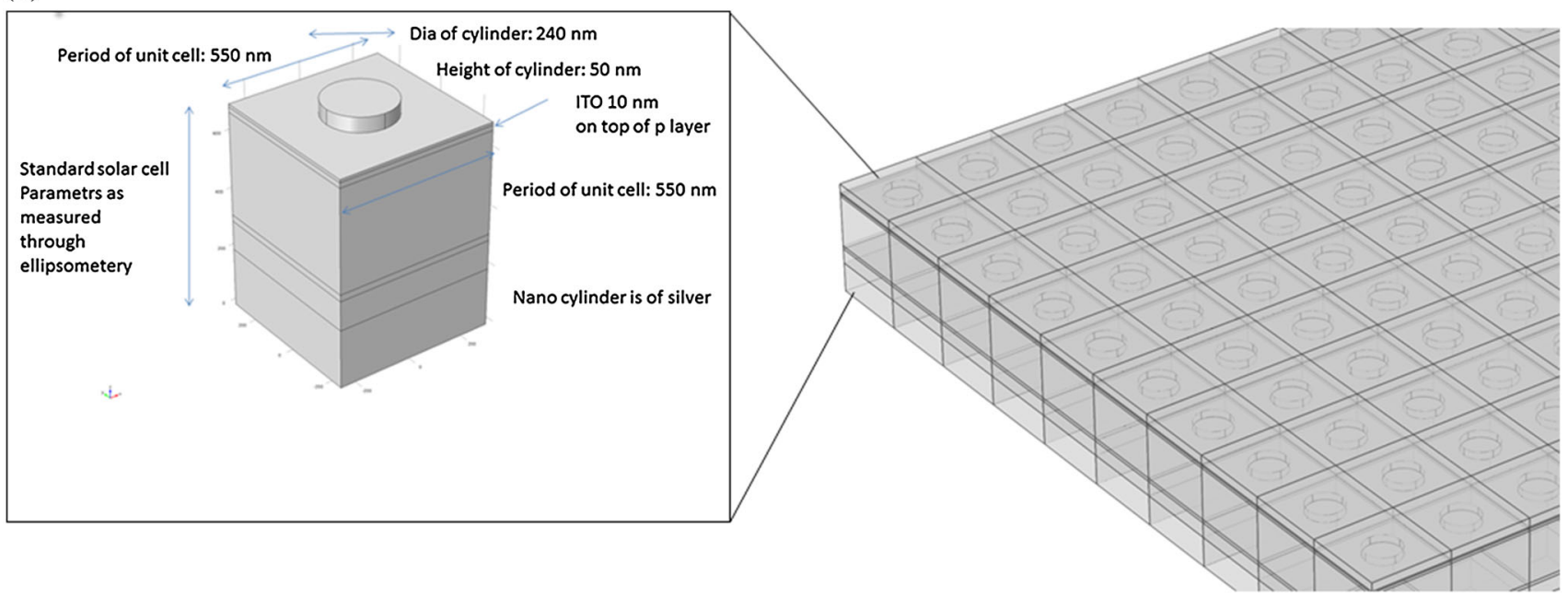

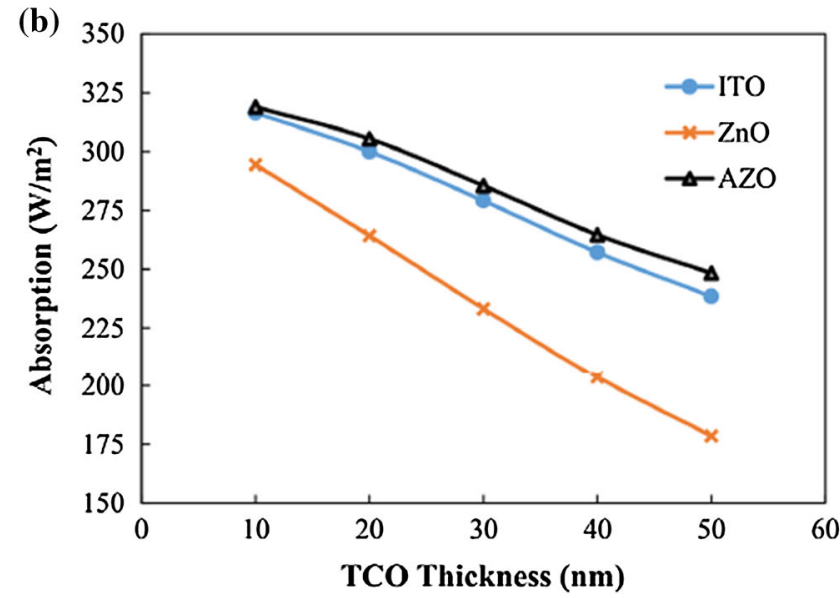

Fig. 1 a Structure of the NDPSC with an enlarged unit cell, b absorption as a function of ITO, $\mathrm{ZnO}$ and AZO thickness. The results simulated using COMSOL show how the useful optical

[30-32]. A $99.99 \%$ 4-inch pressed ITO $\left(\mathrm{Sn}_{2} \mathrm{O}: \operatorname{In}_{2} \mathrm{O}_{3}\right.$ 10:90 \% wt) target was used, and an average base pressure of $7 \times 10^{-8}$ torr was achieved before deposition. Both the glass and silicon substrates were ultrasonically cleaned in isopropanol for $5 \mathrm{~min}$. All other process parameters such as target bias [900 V (ITO and $\mathrm{ZnO}$ ) and $-500 \mathrm{~V}(\mathrm{AZO})$ ] and substrate distance $(75 \mathrm{~mm})$ were kept constant through the experiment. Substrates and target were sputter pre-cleaned in an argon environment for $5 \mathrm{~min}$ before each run. The protocol for pre-cleaning is described in Ref. [29]. To investigate substrate dependency, ITO was deposited on a pair of substrates for 1 min with $0 \%$ oxygen ratio and $100 \mathrm{~W}$ rf power. $\mathrm{ZnO}$ samples were processed at rf power of $100 \mathrm{~W}$ on glass and silicon substrates in an argon environment and $0 \%$ oxygen in the same system as ITO using a stoichiometric $99.99 \%$ 4-inch pressed $\mathrm{ZnO}$ target. The process pressure was maintained at $7.1 \times 10^{-3}$ torr and the deposition rate was calculated to be $8 \mathrm{~nm} / \mathrm{min}$. absorption in the active regions of plasmonic PV devices varies with TCO type and thickness. Theoretically, at small film thicknesses Ohmic losses decrease and useful optical absorption increases [15]

AZO was processed using a Perkin-Elmer Model 2400-8 J rf sputter deposition system using an 8-inch $(203.2 \mathrm{~mm})$ target. The rf power was kept at $500 \mathrm{~W}$, argon flow rate at $18.0 \mathrm{sccm}$, oxygen rate of $2.0 \mathrm{sccm}$ and process pressure at $7.3 \times 10^{-3}$ torr. The system was initially pumped to a base pressure of $6.0 \times 10^{-8}$ torr. The process parameters are summarized in Table 1.

To investigate the effects of post-processing treatment on both optical and electrical effects, additional samples of ITO films on sodalime glass (SLG) substrates were processed using a different instrument [33] to obtain a pair of film samples with varying thicknesses from 10 to $50 \mathrm{~nm}$ in steps of $10 \mathrm{~nm}$. The system is a four-gun sputtering system with a target to substrate spacing of approximately $4^{\prime \prime}$. An ITO (90\% $\mathrm{In}_{2} \mathrm{O}_{3} / 10 \% \mathrm{SnO}_{2}$ from Lesker) target was used. The material was sputtered using $100 \mathrm{~W}$ rf under $4 \mathrm{mTorr}$ of Ar. Deposition time was varied for film thickness with $36 \mathrm{~s}$ resulting in $10 \mathrm{~nm}(\sim 3 \mathrm{~A} / \mathrm{sec})$. This deposition rate 
Table 1 Summary of process parameters for the TCOs

\begin{tabular}{|c|c|c|c|c|c|c|c|}
\hline \multirow[t]{2}{*}{ Sample name } & \multirow[t]{2}{*}{ TCO } & \multirow[t]{2}{*}{ Substrate type } & \multirow[t]{2}{*}{$\mathrm{RF}$ power $(\mathrm{W})$} & \multirow[t]{2}{*}{ Target bias $(\mathrm{V})$} & \multicolumn{2}{|c|}{ Process gases flow rates $(\mathrm{sccm})$} & \multirow[t]{2}{*}{ Film thickness (nm) } \\
\hline & & & & & $\mathrm{Ar}$ & $\mathrm{O}_{2}$ & \\
\hline $0 \mathrm{~A}$ & ITO & Glass & 100 & 900 & 10 & 0 & 9.55 \\
\hline OB & & $\mathrm{Si} / \mathrm{SiO}_{2}$ & & & & & 10.02 \\
\hline $1 \mathrm{~A}_{1}$ & & Glass & & & & & 19.92 \\
\hline $1 \mathrm{~A}_{2}$ & & $\mathrm{Si}$ & & & & & 19.75 \\
\hline 1B & & Glass & & & & & 10.23 \\
\hline $1 \mathrm{C}$ & & & & & & & 20.01 \\
\hline $1 \mathrm{D}$ & & & & & & & 30.79 \\
\hline $1 \mathrm{E}$ & & & & & & & 39.70 \\
\hline $1 \mathrm{~F}$ & & & & & & & 50.03 \\
\hline $2 \mathrm{~A}_{1}$ & $\mathrm{ZnO}$ & Glass & & & & & 9.51 \\
\hline $2 \mathrm{~A}_{2}$ & & $\mathrm{Si} / \mathrm{SiO}_{2}$ & & & & & 10.05 \\
\hline $2 \mathrm{~B}$ & & & & & & & 20.01 \\
\hline $2 \mathrm{C}$ & & & & & & & 29.72 \\
\hline $2 \mathrm{D}$ & & & & & & & 38.98 \\
\hline $2 \mathrm{E}$ & & & & & & & 48.31 \\
\hline $3 \mathrm{~A}$ & $\mathrm{AZO}$ & Glass & 500 & 500 & 18.0 & 2.0 & 12.16 \\
\hline $3 B$ & & $\mathrm{Si} / \mathrm{SiO}_{2}$ & & & & & 11.93 \\
\hline $3 \mathrm{C}$ & & & & & & & 20.39 \\
\hline $3 \mathrm{D}$ & & & & & & & 30.04 \\
\hline $3 \mathrm{E}$ & & & & & & & 40.63 \\
\hline
\end{tabular}

was determined by depositing for a set amount of time and measuring the resulting film thickness using stylus profilometry (Veeco Dektak 150).

One sample for each as-deposited pair was divided into three samples using a diamond scriber. The three pieces were then annealed separately at $400{ }^{\circ} \mathrm{C}$ for 10,20 and $30 \mathrm{~min}$, respectively, using UHP forming gas (FG) (95\% $\mathrm{N}_{2} / 5 \% \mathrm{H}_{2}$ from Air Gas) in a sealed (by vacuum coupling components) quartz tube inside a tube furnace. The furnace was equilibrated at the heating temperature prior to sample introduction. The samples were placed in the quartz tube; then the tube was purged with FG at $5 \mathrm{scfm}$ for $5 \mathrm{~min}$ - this was approximately four exchanges of tube volume. After purging, the samples were introduced into the hot zone with a vacuum-sealed push rod, and the flow rate was reduced to approximately $150 \mathrm{sccm}$ for the duration of heating. After heating, the samples were removed from the hot zone and cooled by increasing the gas flow. After characterization, the sample previously annealed at $400{ }^{\circ} \mathrm{C}$ for $30 \mathrm{~min}$ was further annealed at $500{ }^{\circ} \mathrm{C}$ for $10 \mathrm{~min}$.

\section{Optical and electrical characterization process}

The film thickness measurements and optical characterization were carried out using spectroscopic ellipsometry (J.A Woollam Co UV-VIS V-VASE with control module
VB-400). In each case, a standard scan was performed ranging from 300 to $1000 \mathrm{~nm}$ in increments of $10 \mathrm{~nm}$ for the $65^{\circ}, 70^{\circ}$ and $75^{\circ}$ incident angles. Random detailed scans were performed for the quality check purposes although they are normally not necessary for isotropic samples. Ellipsometry analysis was performed following the process by Synchkova [24]. Intensity measurements were carried out using the VASE for normal transmission incidence $\left(0^{\circ}\right.$ reflection angle) for the three TCOs on glass substrates for the same wavelength range as above. A baseline scan was obtained for the clean SLG substrate first followed by the main data scans using baseline data. Both the baseline and the data scans were acquired in close successions to minimize errors due to light source intensity fluctuations.

Electrical characterization was performed using a fourpoint probe system consisting of ITO optimized tips consisting of 500 micron tip radii set to $60 \mathrm{~g}$ pressure and an RM3000 test unit from Jandel Engineering Limited, UK. The sheet resistance of the 10 and $20 \mathrm{~nm}$ TCOs on glass and on silicon substrates with a spacer oxide layer was determined by direct measurement for both forward and reverse currents. For each TCO on glass sample, a mean sheet resistance value from three random points was used in the final results whilst a mean of only two points was used for the TCO on Si samples since they were smaller. 
All samples were imaged for film quality and a compositional analysis was done using a Hitachi S4700 field emission scanning electron microscope (FE-SEM). Atomic force microscopy (AFM) was performed using a Veeco Dimension 3000 equipment with cantilever tips (Tap300Al-G) on a 1:1 acquisition aspect ratio. The field of view was $2 \mu \mathrm{m}$ at 512 pixel width and scans performed at a speed of $0.5 \mathrm{~Hz}$. Three randomly selected fields of view were acquired per sample and the analyzed areas were limited near to the center of the sample. Roughness analysis was then performed on a defect-free region.

\section{Theory and calculations}

The theoretical derivations of both the resistivity and attenuation coefficient of the ITO films are highlighted in Sects. 3.1 and 3.2 below to explain the underlying processes contributing to the results reported in this paper.

\section{Resistivity measurements}

Sheet resistance measurement was used to obtain the resistivity:

$R=\frac{\rho L}{t W}=R_{s} \frac{L}{W}$,

where $R$ is the resistance, $R_{s}$ is the sheet resistance, and $L$, $W$ and $t$ are the sheet length, width and thickness, respectively.

As the film thickness is measured, the bulk resistivity $\rho$ (in $\mathrm{ohm} \mathrm{cm}$ ) can be calculated by multiplying the sheet resistance by the film thickness in $\mathrm{cm}$ :

$\rho=R_{s} \times t$

\section{Transmittance}

To determine the true transmittance of the TCOs, it was necessary to perform a correction on the experimental data to compensate for losses due to both surface reflection and absorption due to the glass substrate. It is assumed light passing through the glass substrate undergoes attenuation according to Beer-Lambert's law:

$I_{g}=I_{o} e^{-\alpha_{g} t_{g}}$,

where $I_{0}$ and $I_{g}$ represent the initial incident intensity and intensity through the glass substrate, $\alpha_{g}$ and $t_{g}$ are the attenuation coefficient of the glass and glass thickness, respectively.

The total normalized transmittance, $T$ is given by

$T=1-A-R$

where $A$ and $R$ represent the total absorbance and reflectance, respectively.

\section{Results and discussion}

\section{TCOs characterization}

The transmittance and resistivity measurement results for the TCOs are discussed below.

\section{Transmittance}

Figure 2 below shows how transmittance of the TCOs studied varied within the 300-1000 $\mathrm{nm}$ wavelength range.

Transmittance results support the sensitivity analysis results. For the 20-nm films, AZO has greater than $90 \%$ transmittance for the 300-1000 wavelength range, whilst ITO and $\mathrm{ZnO}$ show an average transmittance greater than 80 and $70 \%$, respectively, in the same spectral range.

\section{Sheet resistance}

The resistivity of the $20 \mathrm{~nm}$ as-deposited TCO films on SLG substrates are shown in Table 2. $\mathrm{ZnO}$, despite having the worst transmittance (Fig. 2), has the lowest resistivity among the three TCOs being compared here and AZO has the highest resistivity value. ITO has transmittance comparable to that of AZO and its resistivity is slightly higher than that of $\mathrm{ZnO}$, making it the most promising candidate material for plasmonic-based devices.

Table 3 shows the dependence of ITO sheet resistance with substrate type and thickness. There was a marked difference between the readings on the 10- and 20-nm Si samples; however, there was no discernible difference between the readings on the 10 and $20 \mathrm{~nm}$ on glass. There were very small amounts of fluctuation which can be expected on high resistance samples, and it was more prominent on the Si samples. The readings reversed well, indicating that the film was uniform, with the worst correlation on the 10-nm Si sample. This is the limit of fourpoint probe capability. The 10-nm ITO on glass showed the

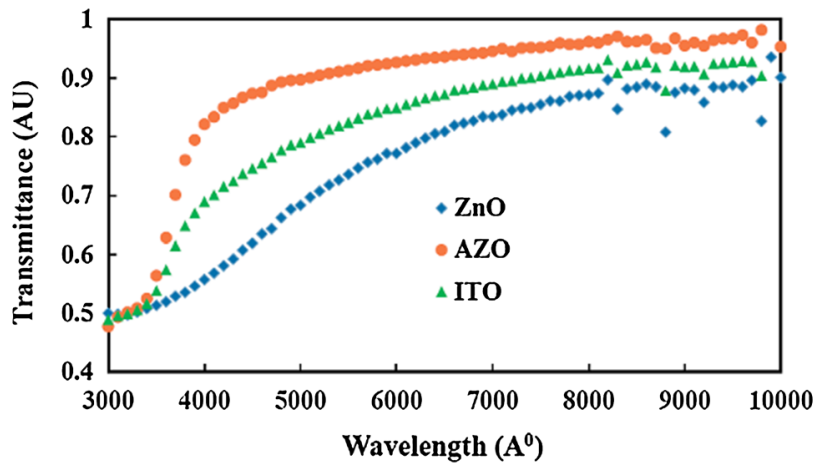

Fig. 2 Transmittance results for $20 \mathrm{~nm}$ thick ITO, $\mathrm{ZnO}$ and $\mathrm{AZO}$ films 
Table 2 Resistivity of $20 \mathrm{~nm}$ as-deposited ITO, AZO and ZnO films on SLG substrates

\begin{tabular}{lllll}
\hline Sample & Substrate & Thickness $(\mathrm{nm})$ & Sheet resistance, $R_{\mathrm{s}}(\Omega / \square) \times 10^{3}$ & Resisitivity, $\rho(\Omega \mathrm{cm})$ \\
\hline ITO & Glass & 20 & 623 & $1.3 \times 10^{-3}$ \\
AZO & Glass & 20 & 876 & $1.7 \times 10^{-3}$ \\
ZnO & Glass & 20 & 390 & $7.8 \times 10^{-4}$ \\
\hline
\end{tabular}

Table 3 Sheet resistance of various as-deposited TCO samples

\begin{tabular}{llllll}
\hline Sample & Substrate & Thickness $(\mathrm{nm})$ & Input current & Sheet resistance, $R_{\mathrm{s}}(\Omega / \square) \times 10^{3}$ & Resisitivity, $\rho(\Omega \mathrm{cm})$ \\
\hline ITO & Glass & 10 & $100 \mathrm{nA}$ & 830 & $8.3 \times 10^{-4}$ \\
& & 20 & $1 \mu \mathrm{A}$ & 623 & $1.3 \times 10^{-3}$ \\
& & & & 422 & $4.2 \times 10^{-4}$ \\
& & & & 83.9 & $1.7 \times 10^{-4}$ \\
\hline
\end{tabular}

highest resistivity whilst the lowest resistivity value was recorded for the 20-nm Si substrate sample. The results are further confirmed by the nature of the microstructure observed by SEM (vide infra) for these samples.

\section{ITO characterization}

\section{Transmittance measurements for ITO}

Transmittance measurements for ITO samples deposited on SLG substrates are shown in Fig. 3. All transmittance values were normalized as given in Eq. (4). It can be noted that there is no discernible difference between the as-deposited and the heat-treated samples particularly for the 30-, 40- and 50-nm films. However, it is also interesting to note that for the 10- and 20-nm films, the as-deposited films have the highest transmittance with the 10-nm film being almost $100 \%$ transmitting throughout the visible spectra. For the $40-\mathrm{nm}$ film, annealing at $400{ }^{\circ} \mathrm{C}$ for 20 min gives the best transmittance. Generally it is observed that heattreated ITO films in FG environment improve transmittance in the UV region of the spectra.

As-deposited thinner ITO samples (10 and $20 \mathrm{~nm}$ ) have the transmittance greater than $95 \%$. It is interesting to note that the 40-nm film sample does not seem to follow this general trend, particularly the sample annealed for $20 \mathrm{~min}$. This sample film shows the greatest increase in mean roughness (vida infra) when all other films' roughness is decreasing and it also has the best transmission for all the 40-nm film samples. The general trend is that the overall transmittance curve for the as-deposited ITO shifts down with increasing film thickness (i.e., the as-deposited film becomes less transparent with increasing thickness as expected). Around visible spectrum and at higher wavelengths, the transmittance for the as-deposited ITO approaches that of the annealed ITO (i.e., annealing is not much effective here in improving transparency). However, around UV wavelengths the transmittance for the as-deposited ITO shifts down below that of the annealed samples (i.e., at small wavelengths annealing is more effective as the annealed samples are more transparent). This is a well-known phenomenon (Burstein-Mess shift) which is a result of ITO optical band gap shifting towards higher energies when annealed in $\mathrm{FG}$ or $\mathrm{H}_{2}$ gas. This is attributed to increase in carrier concentration and is well documented [25]. In addition, it appears that among the annealed samples, $20 \mathrm{~min}$ gives the optimum transmittance for thicknesses below $50 \mathrm{~nm}$, especially at large wavelengths. There observed trend means that the use of thinner $(10 \mathrm{~nm})$, more transmitting and low loss (Ohmic losses) films will result in more light being coupled into the underlying $\mathrm{i}-\mathrm{a}-\mathrm{Si}: \mathrm{H}$ layer rather than being absorbed in the TCO layer as is the case with thicker film $(>20 \mathrm{~nm})$.

\section{Electrical characterization}

Figure 4 shows the dependence of sheet resistance on film thickness, annealing temperature and time. Films annealed for $20 \mathrm{~min}$ give the lowest resistivity and show the same trend as those annealed for $30 \mathrm{~min}$ whilst the as-deposited resistivity versus thickness trend is similar to films annealed for $10 \mathrm{~min}$. Results here show that annealing in FG lowers the resistivity. The lowest resistivity of approximately $4 \times 10^{-4} \Omega \mathrm{cm}$ is for the $40-\mathrm{nm}$ film annealed for $20 \mathrm{~min}$. The highest resistivity value for the annealed samples is for the 20-nm film annealed for $10 \mathrm{~min}$.

\section{Film morphology and roughness}

\section{Effect of substrate on ultra-thin ITO films}

Figure 5 shows results from SEM scans showing the surface morphology for both 10 and $20 \mathrm{~nm}$ as-deposited ITO 

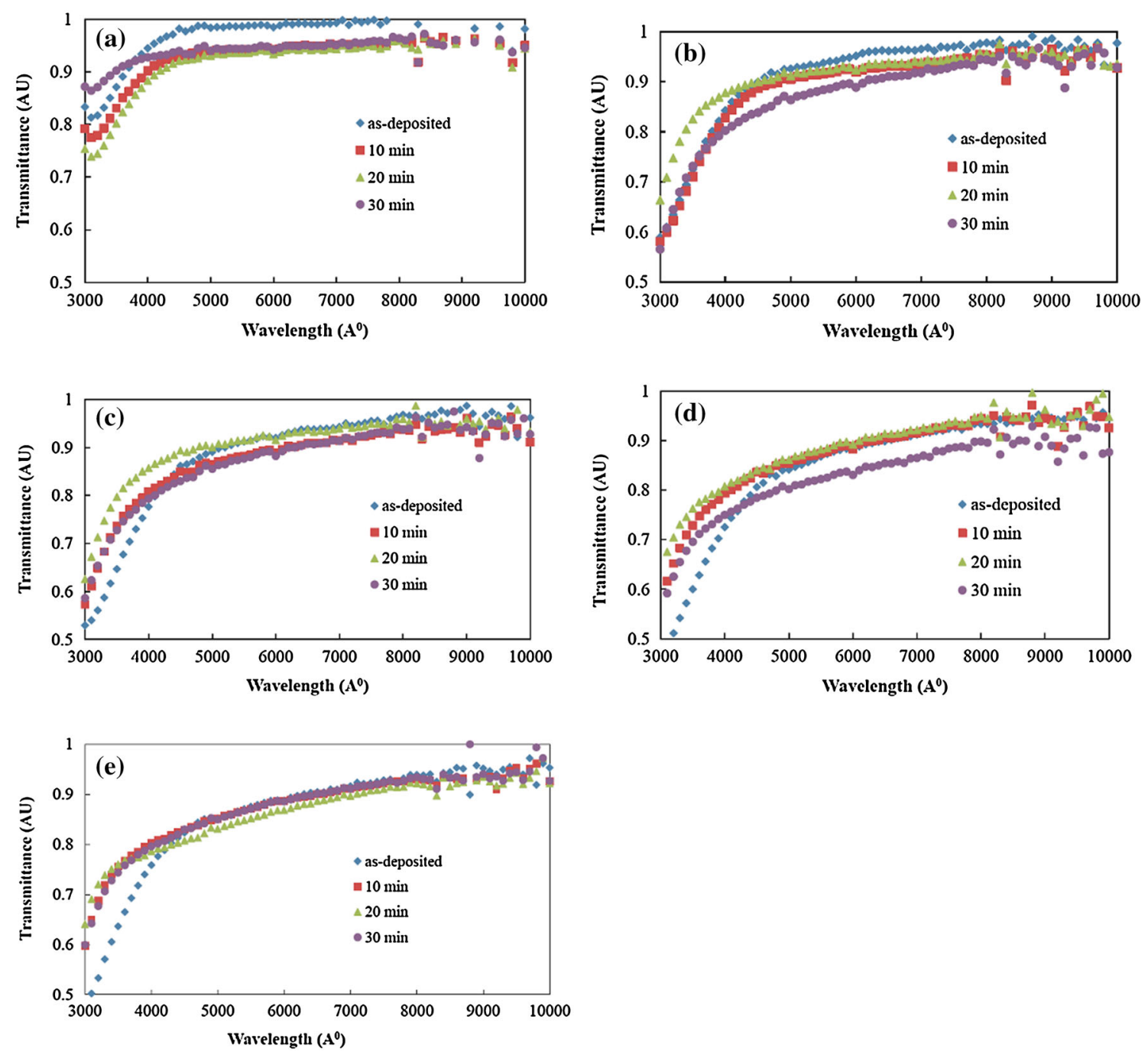

Fig. 3 Transmittance spectra for ITO as-deposited and annealed films on sodalime glass for a $10 \mathrm{~nm}, \mathbf{b} 20 \mathrm{~nm}$, c $30 \mathrm{~nm}$, d $40 \mathrm{~nm}$ and e $50 \mathrm{~nm}$ ITO thickness

films. Figure 5a-c shows that the film surface is relatively smooth and predominantly amorphous in nature. Figure $5 \mathrm{~d}$ shows signs of grains development. The AFM analysis results are shown in Fig. 6.

The results in Fig. 6 show how mean roughness values of ITO vary with substrate type and are in agreement with the results shown in Fig. 5. It can be observed in these images that ITO tends to form uniform features on silicon with no evidence of defects. This is not the case with ITO on glass substrate which, despite having finer features (10 nm film) exhibits some larger defects. These defects seem to increase with the increase in film mean roughness and thickness. Despite the presence of a few dust particles on the sample surface, results confirmed that the sputtered films were of good quality. The AFM roughness results are summarized in Table 4.

\section{Effect of annealing time on ultra-thin ITO films}

When ultra-thin ITO films were subjected to post-processing treatment at $400{ }^{\circ} \mathrm{C}$ in a $\mathrm{FG}$ environment, different treatment times produced different effects.

The as-deposited films mean roughness for this second batch of ITO samples were observed to vary between 0.67 and $0.85 \mathrm{~nm}$. The $10-\mathrm{nm}$ film had the smallest mean roughness value whilst the $20-\mathrm{nm}$ film had the largest value. This may be due to the presence of surface defect features which seem to be more pronounced on the $20-\mathrm{nm}$ 

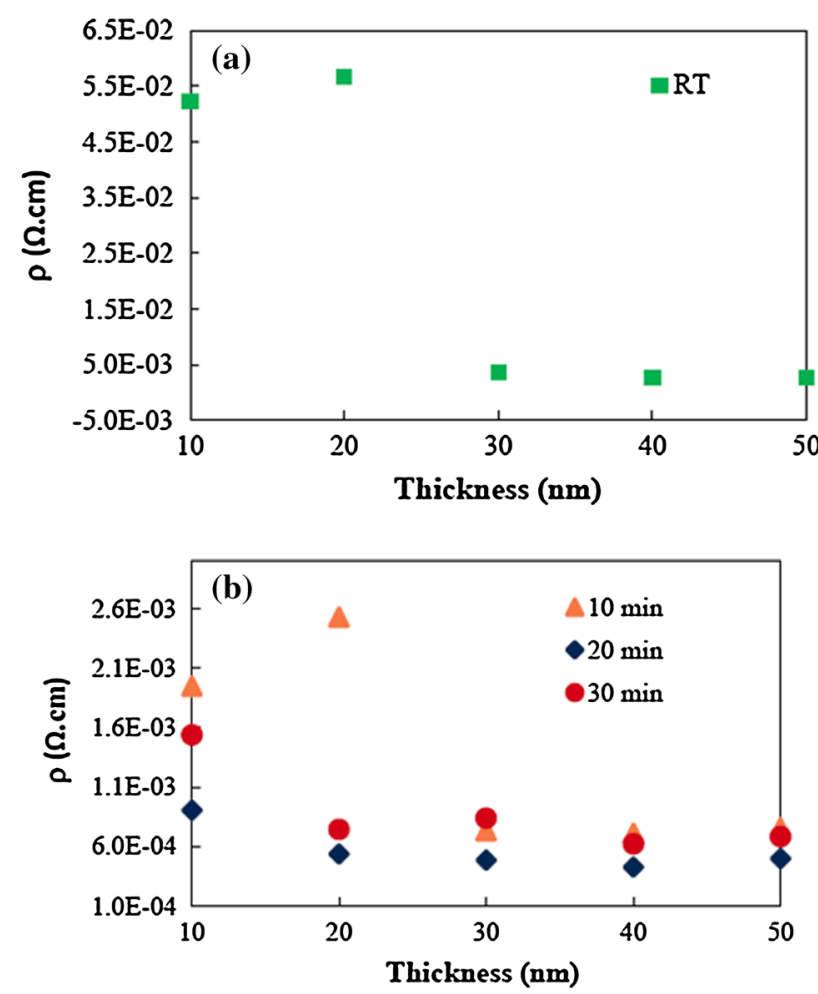

Fig. 4 Variation of resistivity with ITO film thickness for (a) asdeposited/room temperature (RT) (b) annealed films for 10, 20 and $30 \mathrm{~min}$

film compared to all the other samples. Generally, all samples show a varying degree of dust particles' presence and potential artifacts. Sections of the film samples which exhibited heavy dust particles' (and any other contaminants) presence, striations and potential artifacts that were not consistent with other areas on the sample were excluded from the analysis.

The images show a sharp increase in the mean roughness for generally all films after 10 min of heat treatment. Whilst the film roughness is small for both the 10- and 20-nm films, it is observed to increase by a factor of two for the 30- to $50-\mathrm{nm}$ film thickness samples. There is a trend for all films showing a decrease in mean roughness after $20 \mathrm{~min}$ of post-processing treatment with the 30-nm film showing the greatest decrease from approximately 1.9 to $1 \mathrm{~nm}$. Evidently, annealing for $30 \mathrm{~min}$ results in a slight improvement in film roughness for the 30 - to $50-\mathrm{nm}$ range of film. However, the thinner films (10 and $20 \mathrm{~nm})$ show great deterioration in film mean roughness when annealed for longer periods of time (30 min or greater). This can be explained by the onset of islands on both of these films. Island formation is more pronounced on the quasi $2 \mathrm{D}$ 10-nm film resulting in the mean roughness increasing from the initial value of approximately $0.7-1.9 \mathrm{~nm}$.

The effects of annealing different ITO films in forming gas at $400{ }^{\circ} \mathrm{C}$ for 10,20 and $30 \mathrm{~min}$ on films surface roughness are compared and summarized in Fig. 7.

The detailed study on ITO showed some dependency of electrical properties and surface roughness with substrate type which is consistent with results from previous studies on slightly thicker films. Also ITO films on glass show a high degree of surface defects and finer amorphous-like features which may explain the high and oscillating values of sheet resistance on these films. Films grown on $\mathrm{Si}$ substrate have uniform, but large features. However, the same films have higher resistivity values. All samples, asdeposited and annealed, have a transmittance value greater than $80 \%$ with the as-deposited films being superior except for the 40-nm films for which the annealing for 20 min gives the best transmittance. Further analysis of samples shows films annealed for 20 min generally have the lowest resistivity and lower roughness values.

Future work is needed to improve other TCOs such as $\mathrm{AZO}$ and $\mathrm{ZnO}$ and to engineer new high-conductivity lowloss materials for integration into plasmonic devices. AZO exhibited a transmittance superior to that of ITO while $\mathrm{ZnO}$ had the best sheet resistance among the three TCOs being compared. Further investigative work is needed to find the balance between films with useful resistivity and acceptable Ohmic losses in plasmonic-based PV devices. Future work should focus on different processing techniques such as DC sputtering as well as exploring other post-processing environments.

\section{Conclusions}

Ultra-thin TCOs and in particular ITO present a number of challenges for use as thin top contacts on plasmonic-enhanced PV devices. First, both ultra-thin TCO optical and electrical parameters differ greatly from those of thicker (bulk) films deposited under the same conditions. Second, they are delicate due to their thickness, requiring very long annealing times to prevent film cracking. The reactive gases (usually oxygen or hydrogen) require careful monitoring to avoid over-oxidizing or over-reducing the film as it impacts their stoichiometry. There is a trade-off between conductivity and transparency of the deposited films. The sub $50 \mathrm{~nm}$ thick TCO films investigated exhibited desirable optical properties (transmittance greater than $80 \%$ ), which makes them viable for plasmonic PV devices 

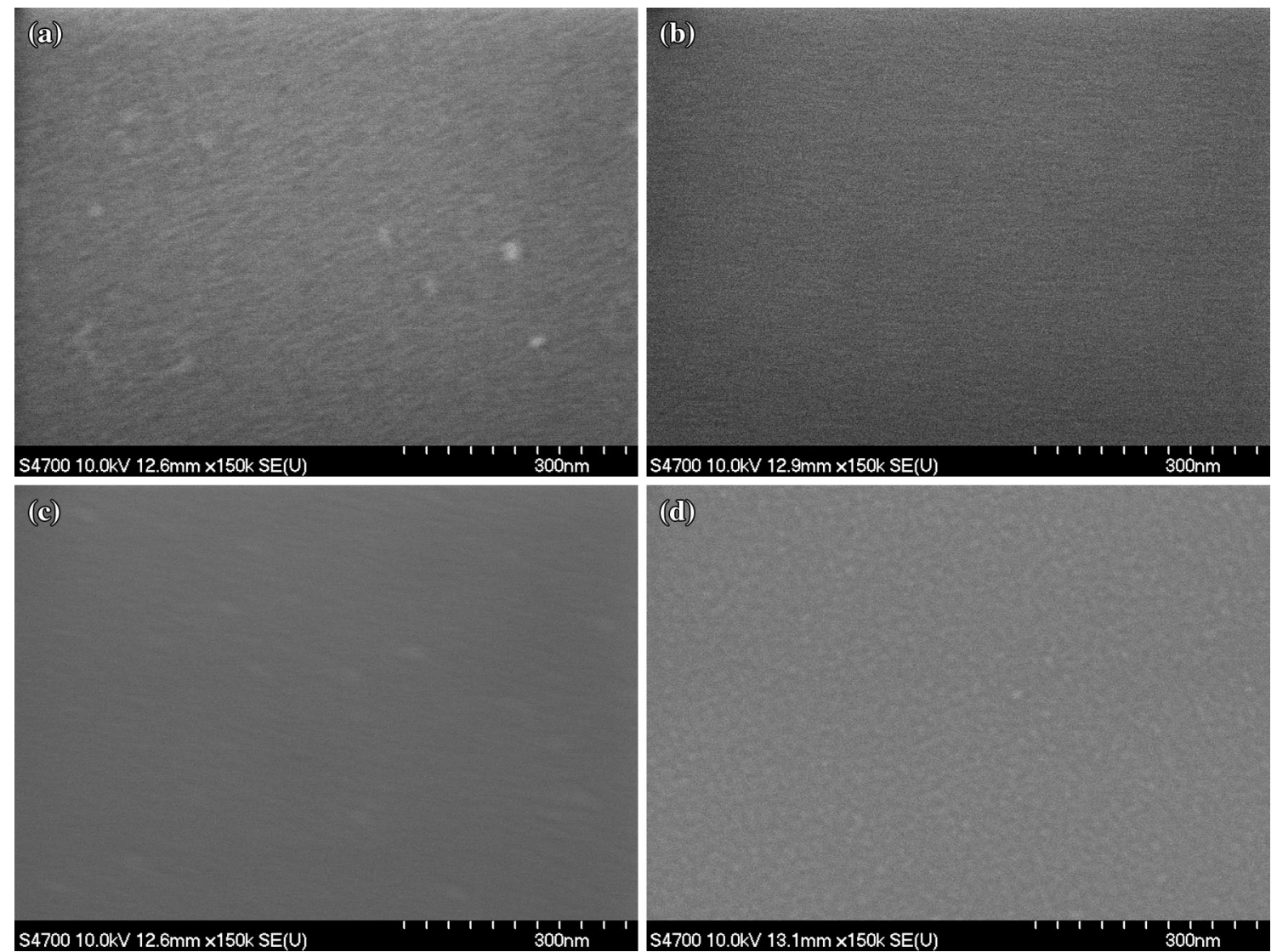

Fig. 5 FESEM images for (a) $10 \mathrm{~nm}$ ITO on glass, (b) $10 \mathrm{~nm}$ ITO on silicon (with oxide spacer), (c) $20 \mathrm{~nm}$ ITO on glass and (d) $20 \mathrm{~nm}$ ITO on silicon (with oxide spacer)

Fig. 6 AFM images for asdeposited (a) $10 \mathrm{~nm}$ ITO on glass, (b) $10 \mathrm{~nm}$ ITO on silicon (with oxide spacer), (c) $20 \mathrm{~nm}$ ITO on glass and (d) $20 \mathrm{~nm}$ ITO on silicon (with oxide spacer).

Image scale is $10 \mathrm{~nm}$
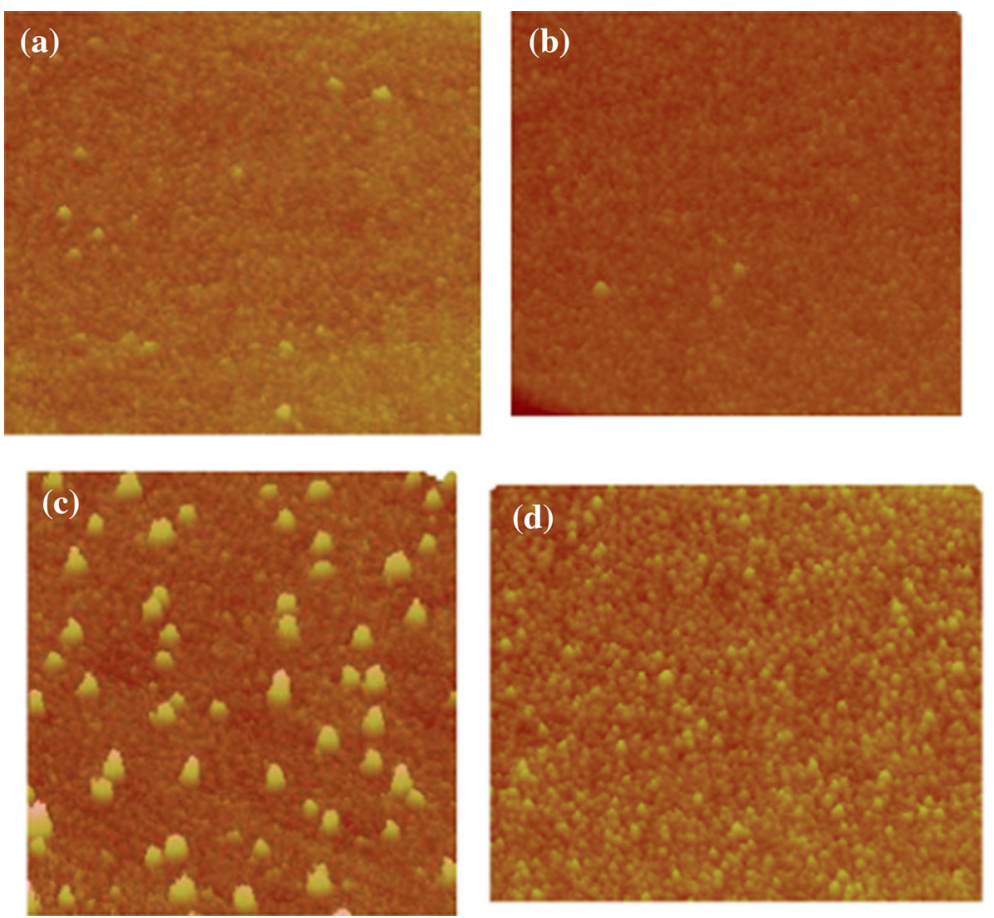

$10.0 \mathrm{~nm}$

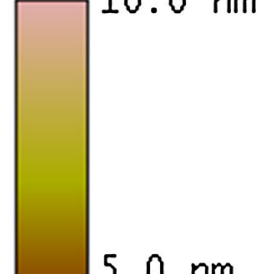

$0.0 \mathrm{~nm}$ 
Table 4 Summary of AFM results for as-deposited ITO films on glass and Si substrates

\begin{tabular}{llll}
\hline Film & Roughness $(\mathrm{nm})$ & Observations \\
\cline { 2 - 4 } & RMS roughness $(\mathrm{Rq})$ & Mean roughness $(\mathrm{Ra})$ & \\
\hline ITO on Si wafer, 10 nm & 0.44 & 0.35 & Uniform, small features \\
ITO on Si wafer, 20 nm & 0.58 & 0.45 & Uniform features larger than 10 nm \\
ITO on glass, 10 nm & 0.37 & 0.29 & Very fine features with some larger defects \\
ITO on glass, 20 nm & 0.83 & 0.52 & Fine features with many large defects \\
\hline
\end{tabular}

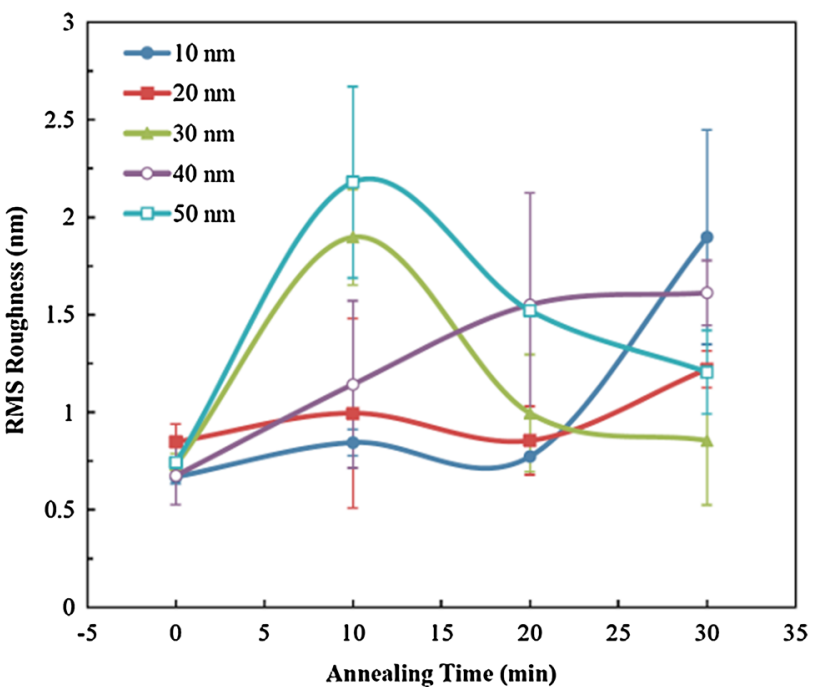

Fig. 7 RMS roughness of annealed ITO films. The figure shows a time series with an overlap of error bars

applications. However, all films evaluated here had resistivity values too high to be considered as materials for the top contact of conventional PV devices.

Acknowledgments Authors would like to acknowledge the support from faculty start-up funds from the University of Toledo, Fulbright (Science and Technology) funds, and the National Science Foundation (CBET-1235750). Furthermore, authors would like to acknowledge the helpful discussion with Dr. J. Mayandi.

Open Access This article is distributed under the terms of the Creative Commons Attribution 4.0 International License (http:// creativecommons.org/licenses/by/4.0/), which permits unrestricted use, distribution, and reproduction in any medium, provided you give appropriate credit to the original author(s) and the source, provide a link to the Creative Commons license, and indicate if changes were made.

\section{References}

1. Shah, A., Torres, P., Tscharner, R., Wyrsch, N., Keppner, H.: Photovoltaic technology: the case for thin-film solar cells. Science 285(5428), 692-698 (1999)

2. Pearce, J.M.: Photovoltaics-a path to sustainable futures. Futures 34(7), 663-674 (2002)
3. Fthenakis, V.: Sustainability of photovoltaics: the case for thinfilm solar cells. Renew Sustain Energy Rev 13(9), 2746-2750 (2009)

4. Saga, T.: Advances in crystalline silicon solar cell technology for industrial mass production. NPG Asia Mater 2(3), 96-102 (2010)

5. Branker, K., Pathak, M.J.M., Pearce, J.M.: A review of solar photovoltaic levelized cost of electricity. Renew Sustain Energy Rev 15(9), 4470-4482 (2011)

6. Green, M.A.: Thin-film solar cells: review of materials, technologies and commercial status. J Mater Sci Mater Electron 18(1), 15-19 (2007)

7. Gwamuri, J., Güney, D.Ö., Pearce, J.M.: Advances in plasmonic light trapping in thin-film solar photovoltaic devices. In: Tiwari, A., Boukherroub, R., Sharon, M. (eds.) Solar cell nanotechnology, pp. 243-270. Wiley, Hoboken (2013)

8. Vora, A., Gwamuri, J., Pala, N., Kulkarni, A., Pearce, J.M., Güney, D.Ö.: Exchanging ohmic losses in metamaterial absorbers with useful optical absorption for photovoltaics. Sci Rep 4:4901 (2014)

9. Khaleque, T., Magnusson, R.: Light management through guidedmode resonances in thin-film silicon solar cells. J Nanophoton 8(1), 083995 (2014)

10. Green, M.A.: Third generation photovoltaics: advanced solar energy conversion (Springer Series in Photonics). Springer (2005)

11. Atwater, H.A., Polman, A.: Plasmonics for improved photovoltaic devices. Nat Mater 9(3), 205-213 (2010)

12. Maier, S.A.: Plasmonics: fundamentals and applications. Springer, New York (2007)

13. Maier, S.A., Atwater, H.A.: Plasmonics: localization and guiding of electromagnetic energy in metal/dielectric structures. J Appl Phys 98(1), 011101 (2005)

14. McPeak, K.M., Jayanti, S.V., Kress, S.J.P., Iotti, S., Rossinelli, A., Norris, D.J.: Plasmonic films can easily be better: rules and recipes. ACS Photon 2(3), 326-333 (2015)

15. Vora, A., Gwamuri, J., Pearce, J.M., Bergstrom, P.L., Güney, D.Ö.: Multi-resonant silver nano-disk patterned thin film hydrogenated amorphous silicon solar cells for Staebler-Wronski effect compensation. J Appl Phys 116(9), 093103 (2014)

16. Kulkarni, A.K., Schulz, K.H., Lim, T.-S., Khan, M.: Electrical, optical and structural characteristics of indium-tin-oxide thin films deposited on glass and polymer substrates. Thin Solid Films 308, 1-7 (1997)

17. Kulkarni, A.K., Schulz, K.H., Lim, T.S., Khan, M.: Dependence of the sheet resistance of indium-tin-oxide thin films on grain size and grain orientation determined from X-ray diffraction techniques. Thin Solid Films 345(2), 273-277 (1999)

18. Leem, J.W., Yu, J.S.: Indium tin oxide subwavelength nanostructures with surface antireflection and superhydrophilicity for high-efficiency Si-based thin film solar cells. Opt. Express 20(103), A431-A440 (2012)

19. Guillen, C., Herrero, J.: Comparison study of ITO thin films deposited by sputtering at room temperature onto polymer and glass substrates. Thin Solid Films 480, 129-132 (2005) 
20. George, J., Menon, C.S.: Electrical and optical properties of electron beam evaporated ITO thin films. Surf. Coat. Technol. 132(1), 45-48 (2000)

21. Alam, M.J., Cameron, D.C.: Characterization of transparent conductive ITO thin films deposited on titanium dioxide film by a sol-gel process. Surf Coat Technol 142, 776-780 (2001)

22. Houng, B., Wang, A.: Characterization of indium tin oxide films by RF-assisted DC magnetron sputtering. Appl. Surf. Sci. 258(15), 5593-5598 (2012)

23. Thestrup, B., Schou, J., Nordskov, A., Larsen, N.B.: Electrical and optical properties of thin indium tin oxide films produced by pulsed laser ablation in oxygen or rare gas atmospheres. Appl Surf Sci 142(1), 248-252 (1999)

24. Terzini, E., Thilakan, P., Minarini, C.: Properties of ITO thin films deposited by RF magnetron sputtering at elevated substrate temperature. Mater Sci Eng B 77(1), 110-114 (2000)

25. Sytchkova, A., Zola, D., Bailey, L.R., Mackenzie, B., Proudfoot, G., Tian, M., Ulyashin, A.: Depth dependent properties of ITO thin films grown by pulsed DC sputtering. Mater Sci Eng B 178(9), 586-592 (2013)

26. Cruz, L., Legnani, C., Matoso, I., Ferreira, C., Moutinho, H.: Influence of pressure and annealing on the microstructural and electro-optical properties of RF magnetron sputtered ITO thin films. Mater Res Bull 39, 993-1003 (2004)
27. Chen, B.J., Sun, X.W., Tay, B.K.: Fabrication of ITO thin films by filtered cathodic vacuum arc deposition. Mater Sci Eng B 106(3), 300-304 (2004)

28. Tseng, K.-S., Lo, Y.-L.: Effect of sputtering parameters on optical and electrical properties of ITO films on PET substrates. Appl Surf Sci 285, 157-166 (2013)

29. Kim, D.-H., Park, M.-R., Lee, H.-J., Lee, G.-H.: Thickness dependence of electrical properties of ITO film deposited on a plastic substrate by RF magnetron sputtering. Appl Surf Sci 253(2), 409-411 (2006)

30. Betz, U., Olsson, M.K., Marthy, J., Escolá, M.F.: On the synthesis of ultra smooth ITO thin films by conventional direct current magnetron sputtering. Thin Solid Films 516(7), 1334-1340 (2008)

31. Tahar, R.B.H., Ban, T., Ohya, Y., Takahashi, Y.: Tin doped indium oxide thin films: electrical properties. J. Appl. Phys. 83(5), 2631-2645 (1998)

32. Perkin-Elmer, R.F.: Sputtering System-6 Inch protocol: MOST. http://www.appropedia.org/Perkin-Elmer_RF_Sputtering_System6_Inch_protocol:_MOST. Accessed 10 Jul 2015

33. Knickerbocker, S.A.: Ph.D. Dissertation, Michigan Technological University (1995) 